\title{
A!
}

This is an electronic reprint of the original article.

This reprint may differ from the original in pagination and typographic detail.

VERITAS Collaboration; SPOL; ASAS-SN; OVRO; NuSTAR Team; CRTS

Gamma-Rays from the Quasar PKS 1441+25

Published in:

Astrophysical Journal Letters

DOI:

10.1088/2041-8205/815/2/L22

Published: 01/01/2015

Document Version

Publisher's PDF, also known as Version of record

Please cite the original version:

VERITAS Collaboration, SPOL, ASAS-SN, OVRO, NuSTAR Team, \& CRTS (2015). Gamma-Rays from the Quasar PKS 1441+25: Story of an Escape. Astrophysical Journal Letters, 815(2), L22.

https://doi.org/10.1088/2041-8205/815/2/L22

This material is protected by copyright and other intellectual property rights, and duplication or sale of all or part of any of the repository collections is not permitted, except that material may be duplicated by you for your research use or educational purposes in electronic or print form. You must obtain permission for any other use. Electronic or print copies may not be offered, whether for sale or otherwise to anyone who is not an authorised user. 


\section{GAMMA-RAYS FROM THE QUASAR PKS 1441+25: STORY OF AN ESCAPE}

A. U. Abeysekara ${ }^{1}$, S. Archambault ${ }^{2}$, A. Archer ${ }^{3}$, T. Aune ${ }^{4}$, A. Barnacka ${ }^{5}$, W. Benbow ${ }^{6}$, R. Bird ${ }^{7}$ J. Biteau $^{8,9}$,

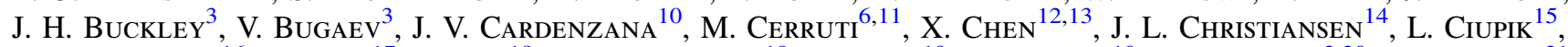
M. P. Connolly ${ }^{16}$, P. Coppi ${ }^{17}$, W. Cui ${ }^{18}$, H. J. Dickinson ${ }^{19}$, J. Dumm ${ }^{19}$, J. D. Eisch ${ }^{10}$, M. Errando ${ }^{3,20}$, A. Falcone ${ }^{21}$, Q. Feng ${ }^{18}$, J. P. Finley ${ }^{18}$, H. Fleischhack ${ }^{19}$, A. Flinders ${ }^{1}$, P. Fortin ${ }^{6}$, L. Fortson ${ }^{19}$, A. Furniss ${ }^{8,22}$, G. H. Gillanders ${ }^{16}$,

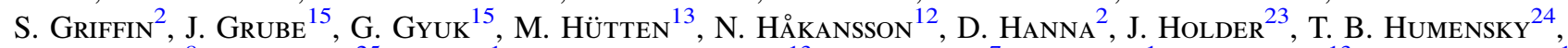
C. A. Johnson ${ }^{8}$, P. KaARet ${ }^{25}$, P. Kar ${ }^{1}$, N. Kelley-Hoskins ${ }^{13}$, Y. Khassen ${ }^{7}$, D. Kieda ${ }^{1}$, M. Krause ${ }^{13}$, F. Krennrich ${ }^{10}$, S. Kumar ${ }^{23}$, M. J. Lang ${ }^{16}$, G. Maier ${ }^{13}$, S. McArthur ${ }^{18}$, A. McCann ${ }^{2}$, K. Meagher ${ }^{26}$, P. Moriarty ${ }^{16}$, R. Mukherjee ${ }^{20}$, D. Nieto ${ }^{24}$, A. O’FaOláin de Bhróithe ${ }^{13}$, R. A. Ong ${ }^{4}$, A. N. Otte ${ }^{26}$, N. PARK ${ }^{27}$, J. S. Perkins ${ }^{28}$, A. PetrashyK ${ }^{24}$, M. Pohl ${ }^{12,13}$,

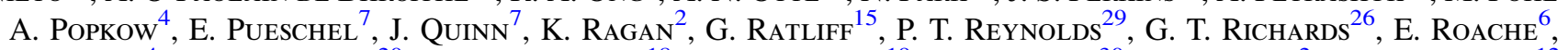
J. Rousselle ${ }^{4}$, M. Santander ${ }^{20}$, G. H. Sembroski ${ }^{18}$, K. Shahinyan ${ }^{19}$, A. W. Smith ${ }^{30}$, D. StaszaK ${ }^{2}$, I. Telezhinsky ${ }^{12,13}$, N. W. Todo ${ }^{3}$, J. V. Tucci ${ }^{18}$, J. Tyler ${ }^{2}$, V. V. VAssiliev ${ }^{4}$, S. Vincent $^{13}$, S. P. WAKely 27 , O. M. Weiner ${ }^{24}$, A. Weinstein ${ }^{10}$, A. Wilhelm ${ }^{12,13}$, D. A. Williams ${ }^{8}$, B. Zitzer ${ }^{31}$

$$
\begin{aligned}
& \text { (VERITAS), } \\
& \text { P. S. SMITH }{ }^{32} \\
& \text { (SPOL), }
\end{aligned}
$$

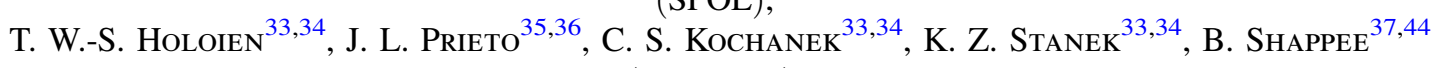

(ASAS-SN),

T. Hovatta ${ }^{38}$, W. MaX-Moerbeck ${ }^{39}$, T. J. Pearson ${ }^{40}$, R. A. Reeves ${ }^{41}$, J. L. Richards ${ }^{18}$, A. C. S. Readhead ${ }^{40}$ (OVRO),

G. M. MADEJSKI ${ }^{42}$

(NUSTAR),

AND

S. G. Djorgovski ${ }^{43}$, A. J. Drake ${ }^{43}$, M. J. Graham ${ }^{43}$, and A. Mahabal ${ }^{43}$ (CRTS)

${ }^{1}$ Department of Physics and Astronomy, University of Utah, Salt Lake City, UT 84112, USA

${ }_{2}^{2}$ Physics Department, McGill University, Montreal, QC H3A 2T8, Canada

${ }^{3}$ Department of Physics, Washington University, St. Louis, MO 63130, USA

${ }^{4}$ Department of Physics and Astronomy, University of California, Los Angeles, CA 90095, USA

${ }^{5}$ Harvard-Smithsonian Center for Astrophysics, 60 Garden Street, Cambridge, MA 02138, USA

${ }^{6}$ Fred Lawrence Whipple Observatory, Harvard-Smithsonian Center for Astrophysics, Amado, AZ 85645, USA; matteo.cerruti@cfa.harvard.edu 7 School of Physics, University College Dublin, Belfield, Dublin 4, Ireland

${ }^{8}$ Santa Cruz Institute for Particle Physics and Department of Physics, University of California, Santa Cruz, CA 95064, USA; caajohns@ucsc.edu

${ }_{9}^{9}$ Now at Institut de Physique Nucléaire d'Orsay (IPNO), CNRS-IN2P3, Univ. Paris-Sud, Université Paris-Saclay, F-91400 Orsay, France; jbiteau@ucsc.edu, biteau@ipno.in2p3.fr

${ }^{10}$ Department of Physics and Astronomy, Iowa State University, Ames, IA 50011, USA

${ }^{11}$ Now at Sorbonne Universités, UPMC Univ. Paris Diderot, Sorbonne Paris Cité, CNRS, Laboratoire de Physique Nucléaire et de Hautes Energies (LPNHE), 4 place Jussieu, F-75252, Paris Cedex 5, France; mcerruti@1pnhe.in2p3.fr

${ }^{12}$ Institute of Physics and Astronomy, University of Potsdam, D-14476 Potsdam-Golm, Germany

14 DESY, Platanenallee 6, D-15738 Zeuthen, Germany

${ }_{15}^{14}$ Physics Department, California Polytechnic State University, San Luis Obispo, CA 94307, USA

${ }^{15}$ Astronomy Department, Adler Planetarium and Astronomy Museum, Chicago, IL 60605, USA
School of Physics, National University of Ireland Galway, University Road, Galway, Ireland; mark.lang@ @uigalway.ie

${ }^{17}$ Department of Astronomy, Yale University, New Haven, CT 06520-8101, USA

${ }_{19}^{18}$ Department of Physics and Astronomy, Purdue University, West Lafayette, IN 47907, USA

${ }^{19}$ School of Physics and Astronomy, University of Minnesota, Minneapolis, MN 55455, USA

${ }^{20}$ Department of Physics and Astronomy, Barnard College, Columbia University, NY 10027, USA; errando@ astro.columbia.edu

21 Department of Astronomy and Astrophysics, 525 Davey Lab, Pennsylvania State University, University Park, PA 16802, USA

${ }^{22}$ Now at California State University-East Bay, Hayward, CA 94542, USA

${ }^{23}$ Department of Physics and Astronomy and the Bartol Research Institute, University of Delaware, Newark, DE 19716, USA

${ }^{24}$ Physics Department, Columbia University, New York, NY 10027, USA

${ }^{25}$ Department of Physics and Astronomy, University of Iowa, Van Allen Hall, Iowa City, IA 52242, USA

${ }^{26}$ School of Physics and Center for Relativistic Astrophysics, Georgia Institute of Technology, 837 State Street NW, Atlanta, GA 30332-0430, USA

${ }^{27}$ Enrico Fermi Institute, University of Chicago, Chicago, IL 60637, USA

${ }^{28}$ N.A.S.A./Goddard Space-Flight Center, Code 661, Greenbelt, MD 20771, USA

29 Department of Applied Science, Cork Institute of Technology, Bishopstown, Cork, Ireland

${ }^{30}$ University of Maryland, College Park/NASA GSFC, College Park, MD 20742, USA

${ }^{31}$ Argonne National Laboratory, 9700 S. Cass Avenue, Argonne, IL 60439, USA
${ }^{32}$ Steward Observatory, University of Arizona, 933 N. Cherry Avenue, Tucson, AZ 85721, USA

${ }^{33}$ Department of Astronomy, The Ohio State University, 140 West 18th Avenue, Columbus, OH 43210, USA

${ }^{34}$ Center for Cosmology and AstroParticle Physics, The Ohio State University, 191 W. Woodruff Ave., Columbus, OH 43210, USA

${ }^{35}$ Nucleo de Astronomia de la Facultad de Ingenieria, Universidad Diego Portales, Av. Ejercito 441, Santiago, Chile

${ }^{36}$ Millennium Institute of Astrophysics, Santiago, Chile

${ }^{37}$ Carnegie Observatories, 813 Santa Barbara Street, Pasadena, CA 91101, USA

${ }^{38}$ Aalto University, Metsähovi Radio Observatory, Metsähovintie 114, FI-02540, Kylmälä, Finland 


\author{
${ }^{39}$ National Radio Astronomy Observatory, P.O. Box 0, Socorro, NM 87801, USA \\ ${ }^{40}$ Cahill Center for Astronomy and Astrophysics, California Institute of Technology, Pasadena, CA 91125, USA \\ ${ }^{41}$ CePIA, Departamento de Astronomía, Universidad de Concepción, Casilla 160-C, Concepción, Chile \\ ${ }^{42}$ W. W. Hansen Experimental Physics Laboratory, Kavli Institute for Particle Astrophysics and Cosmology, Department of Physics and \\ SLAC National Accelerator Laboratory, Stanford University, Stanford, CA 94305, USA \\ ${ }^{43}$ California Institute of Technology, 1200 E. California Blvd, Pasdena, CA 91125 , USA \\ Received 2015 August 10; accepted 2015 November 17; published 2015 December 15
}

\begin{abstract}
Outbursts from gamma-ray quasars provide insights on the relativistic jets of active galactic nuclei and constraints on the diffuse radiation fields that fill the universe. The detection of significant emission above $100 \mathrm{GeV}$ from a distant quasar would show that some of the radiated gamma-rays escape pair-production interactions with lowenergy photons, be it the extragalactic background light (EBL), or the radiation near the supermassive black hole lying at the jet's base. VERITAS detected gamma-ray emission up to $\sim 200 \mathrm{GeV}$ from PKS $1441+25(z=0.939)$ during 2015 April, a period of high activity across all wavelengths. This observation of PKS $1441+25$ suggests that the emission region is located thousands of Schwarzschild radii away from the black hole. The gamma-ray detection also sets a stringent upper limit on the near-ultraviolet to near-infrared EBL intensity, suggesting that galaxy surveys have resolved most, if not all, of the sources of the EBL at these wavelengths.
\end{abstract}

Key words: cosmology: observations - diffuse radiation - gamma rays: galaxies - quasars: individual (PKS 1441+25=VER J1443+250) - radiation mechanisms: non-thermal

\section{A NEW VERY HIGH ENERGY QUASAR}

The extragalactic gamma-ray sky is dominated by the emission of blazars, active galactic nuclei whose jets are pointed within a few degrees of Earth. About sixty blazars have been detected at very high energy (VHE; $E>100 \mathrm{GeV}$ ), ${ }^{45}$ with only four belonging to the class of flat-spectrum radio quasars (FSRQs): $3 C 279 \quad(z=0.536, \quad$ MAGIC Collaboration et al. 2008), PKS 1510-089 $(z=0.361$, H.E.S.S. Collaboration et al. 2013a), PKS 1222+216 $(z=0.432$, Aleksić et al. 2011), and S3 0218+35 $(z=0.944$, Sitarek et al. 2015).

FSRQs are believed to host radiatively efficient disks that enrich the environment of the supermassive black hole with ultraviolet-to-optical photons. This photon field, the reprocessed emission from the clouds of the broad line region (BLR), and the infrared radiation from the "dusty torus" can all interact with gamma-rays through pair production, preventing the escape of VHE radiation from the base of the jet (Donea \& Protheroe 2003).

VHE gamma-rays that do escape will face pair production on the extragalactic background light (EBL), which encompasses the ultraviolet-to-infrared emission of all stars and galaxies since the epoch of reionization $(z \lesssim 10) .{ }^{46}$ Direct measurements of the EBL are prone to contamination from the bright local environment, while strict lower limits are derived from galaxy surveys, measuring the light emitted by known populations of sources (Madau \& Pozzetti 2000).

VHE detections of high-redshift FSRQs constrain both the EBL and the gamma-ray emission regions in blazars. This letter reports the detection of VHE gamma-rays from the FSRQ PKS $1441+25(z=0.939$, Shaw et al. 2012) by VERITAS. This source was observed in 2015 April and May following the VHE discovery by MAGIC (Mirzoyan 2015), triggered by multiwavelength activity (Pacciani 2015) and a spectral hardening at high energies (HE, $100 \mathrm{MeV}<E<100 \mathrm{GeV}$; D. Thompson 2015, private communication on behalf of the Fermi-LAT team).

\footnotetext{
${ }^{44}$ Hubble, Carnegie-Princeton Fellow.

45 http://tevcat.uchicago.edu/

${ }^{46}$ We adopt the concordance $\Lambda \mathrm{CDM}$ model $\left(h_{0}=0.7, \Omega_{M}=0.3, \Omega_{\Lambda}=0.7\right)$.
}

\section{OBSERVATIONS OF PKS $1441+25$}

PKS 1441+25 was detected from 2015 April 21 (MJD 57133) to April 28 (MJD 57140) with VERITAS, an array of four imaging atmospheric Cherenkov telescopes located in southern Arizona (Holder 2011). VERITAS imaged gamma-ray induced showers from the source above $80 \mathrm{GeV}$, enabling the detection of PKS 1441+25 (VER J1443+250) at a position consistent with its radio location and at a significance of 7.7 standard deviations $(\sigma)$ during the $15.0 \mathrm{hr}$ exposure (2710 ON-source events, 13780 OFF-source events, OFF normalization of $1 / 6$ ). Using a standard analysis with cuts optimized for low-energy showers (Archambault et al. 2014, and references therein), we measure an average flux of $\Phi(>80 \mathrm{GeV})=(5.0 \pm 0.7) \times 10^{-11} \mathrm{~cm}^{-2} \mathrm{~s}^{-1}$ with a photon index $\Gamma_{\mathrm{VHE}}=5.3 \pm 0.5$ up to $200 \mathrm{GeV},{ }^{47}$ corresponding to an intrinsic index of $3.4 \pm 0.5$ after correction for the EBL (Gilmore et al. 2012, "fixed"). The day-by-day lightcurve is compatible with constant emission in that period $\left(\chi^{2} /\right.$ $n d f=7.4 / 6$ ), and fractional variability $F_{\text {var }}<110 \%$ at the $95 \%$ confidence level (Vaughan et al. 2003). ${ }^{48}$ Subsequent observations in May (MJD 57155-57166, $3.8 \mathrm{hr}$ exposure) showed no significant excess (660 ON-source events, 3770 OFF-source events, OFF normalization of $1 / 6$ ), resulting in an upper limit of $\Phi(>80 \mathrm{GeV})<4.3 \times 10^{-11} \mathrm{~cm}^{-2} \mathrm{~s}^{-1}$ at the $99 \%$ confidence level. These results have been cross checked with an independent calibration and analysis. Monte-Carlo simulations indicate systematic uncertainties on the VHE energy scale and photon index of $20 \%$ and 0.2 , respectively. The systematic uncertainty on the flux of this source is estimated to be $60 \%$, including the energy-scale uncertainty discussed in Archambault et al. (2014).

The LAT pair-conversion telescope onboard the Fermi satellite has surveyed the whole sky in the HE band since 2008 August (Atwood et al. 2009). We analyzed the LAT data using the public science tools v10r0p5 (Pass-8) leaving free the parameters of sources from the 3FGL (Acero et al. 2015)

\footnotetext{
47 The last spectral points at 140 and $180 \mathrm{GeV}$ are significant at the 2.4 and $3.0 \sigma$ level, respectively.

${ }^{48}$ All flux estimates are used for variability constraints but we also show $99 \%$ confidence-level upper limits for points below $3 \sigma$ in Figure 1.
} 

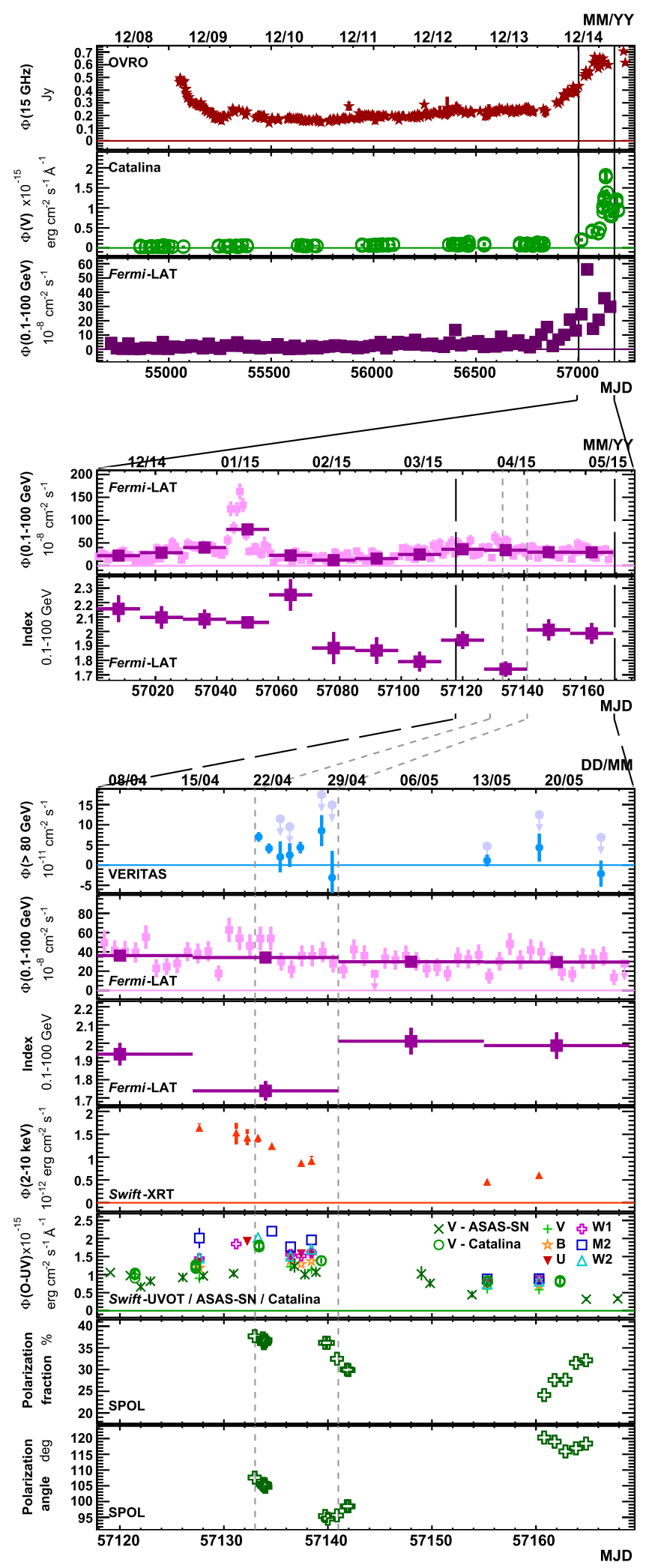

Figure 1. Top: observations of PKS $1441+25$ from 2008 to 2015. Middle: observations from 2014 December to 2015 May. Bottom: observations in April and May. The gray dashed lines mark the period considered for the analyses in Sections 3 and 4 .

within a region of interest of $10^{\circ}$ radius and fixing them for sources $10^{\circ}-20^{\circ}$ away. We reconstruct the spectrum of PKS $1441+25$ between $100 \mathrm{MeV}$ and $100 \mathrm{GeV}$ in four-week
(MJD 54705-57169, top panel in Figure 1) and two-week (MJD 57001-57169, middle panel) bins assuming a power-law model with a free normalization and photon index (purple points), as well as in one-day bins (pink points) fixing the photon index to its best-fit average value in MJD $57001-57169, \Gamma_{\mathrm{HE}}=1.97 \pm 0.02$, slightly harder than in the 3FGL, $2.13 \pm 0.07$. The source was in a high state during MJD 57001-57169, with an integrated $100 \mathrm{MeV}-100 \mathrm{GeV}$ flux that is one to two orders of magnitude above the 3FGL value, $(1.3 \pm 0.1) \times 10^{-8} \mathrm{~cm}^{-2} \mathrm{~s}^{-1}$. During the period contemporaneous with the VERITAS detection (MJD 57133-57140), the source shows a flux of $(34 \pm 4) \times 10^{-8} \mathrm{~cm}^{-2} \mathrm{~s}^{-1}$ and a hard index of $1.75 \pm 0.08$. Although a power-law model is used for robustness in the lightcurve determination, the spectrum shows a hint of curvature, with a log parabola preferred over a power law by $3.2 \sigma$ (see Figure 2 ). The curvature is resilient to changes in the analysis and the temporal window, and fits in smaller energy ranges confirm the hint.

X-ray observations with $N U S T A R$ and Swift were triggered following the VHE detection. NUSTAR, a hard-X-ray instrument sensitive to 3-79 keV photons (Harrison et al. 2013), observed the source on MJD 57137 for an exposure of $38.2 \mathrm{ks}$. The data were reduced using the NuSTARDAS software v1.3.1. Swift-XRT (Gehrels et al. 2004) observed PKS $1441+25$ between 0.3 and $10 \mathrm{keV}$ in 2010 June (MJD 55359), in 2015 January (MJD 57028 and 57050), in 2015 April (MJD 57127-57138), and in 2015 May (MJD 57155 and 57160). Data taken in photon-counting mode were calibrated and cleaned with xrtpipeline using CALDB 20140120 v.014. ON-source and background events were selected within regions of 20-pixel ( $\sim 6$ arcsec) and 40pixel radius, respectively. The XRT and NuSTAR spectral analyses were performed with XPSEC v12.8.2, requiring at least 20 counts per bin. The $N u S T A R$ spectrum is matched by a power law with a photon index of $2.30 \pm 0.10$ and an integrated $3-30 \mathrm{keV}$ flux of $(1.25 \pm 0.09) \times 10^{-12} \mathrm{erg} \mathrm{cm}^{-2} \mathrm{~s}^{-1}$. No intranight variability is detected. Swift-XRT did not significantly detect the source in 2010 June, but the 2015 observations reveal a power-law spectrum with no detectable spectral variability and an average $0.3-10 \mathrm{keV}$ photon index of $2.35 \pm 0.24$, using an absorbed model with a hydrogen column density of $3.19 \times 10^{20} \mathrm{~cm}^{-2}$ (Kalberla et al. 2005). Significant flux variations are detected in the period contemporaneous with VERITAS observations $\left(\chi^{2} / n d f=25.9 / 3, F_{\text {var }}=22.6 \pm 0.9 \%\right)$, with a flux-halving time of $13.9 \pm 1.4$ days based on an exponential fit to the data in MJD 57127-57155 $\left(\chi^{2} /\right.$ $n d f=8.6 / 6)$.

Simultaneously with the XRT observations, Swift-UVOT (Roming et al. 2005) took photometric snapshots of PKS 1441 +25 in six optical-to-ultraviolet filters. Flux densities were extracted using uvotmaghist and circular ON-source and background regions of 5 and 15 arcsec radius, respectively. PKS $1441+25$ has been observed in the $V$-band since 2012 January within the All-Sky Automated Survey for Supernovae (ASAS-SN; Shappee et al. 2014), using the quadruple 14-cm "Brutus" telescope in Hawaii. The fluxes from both experiments in Figure 2 are dereddenned using $E(B-V)=0.043$, consistent with the column density used for the XRT analysis (Jenkins \& Savage 1974). The 0.68-m Catalina Schmidt Telescope (AZ) has also performed long-term unfiltered optical observations of PKS $1441+25$ since 2005 within the Catalina Real-time Transient Survey (CRTS, Drake et al. 2009). 

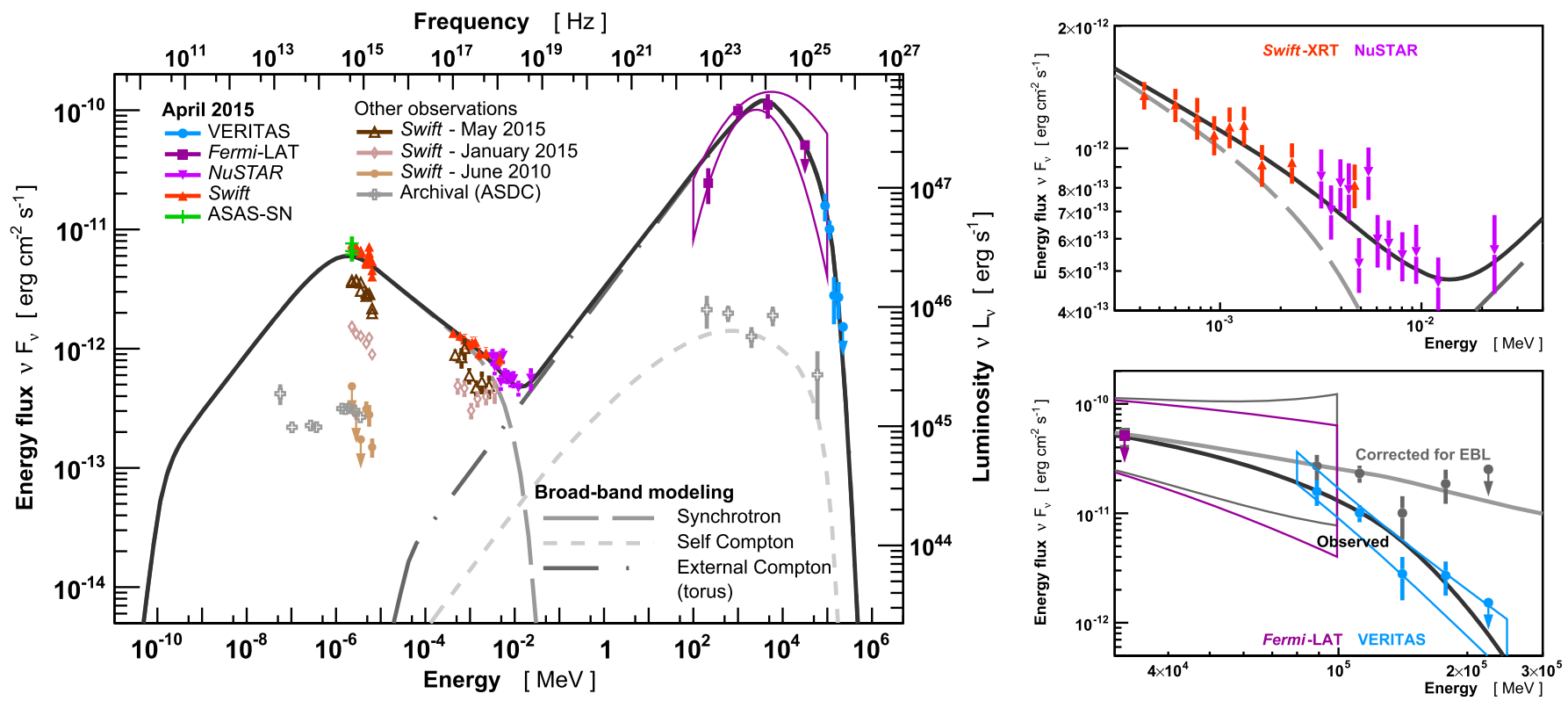

Figure 2. Multiwavelength emission of PKS 1441+25. Side panels show the X-ray (top) and gamma-ray emission (bottom) in 2015 April (MJD 57133-57140). The various exposures and the model are discussed in Sections 2 and 3, respectively.

Observed magnitudes were converted into the $V$-band using the empirical method described in Drake et al. (2013). The SPOL spectropolarimeter (Schmidt et al. 1992) has monitored the linear optical polarization of PKS $1441+25$ in $5000-7000 \AA$, with observations at the 1.54-m Kuiper Telescope, at the $6.5-\mathrm{m}$ MMT, and at the Steward Observatory 2.3-m Bok Telescope (AZ). The source shows a high degree of polarization, with values ranging from $37.7 \pm 0.1 \%$ to $36.2 \pm 0.1 \%$ between MJD 57133 and MJD 57140.

The OVRO 40-m telescope (Richards et al. 2011) has monitored PKS $1441+25$ at $15 \mathrm{GHz}$ since late 2009 . A $15 \mathrm{GHz}$ VLBA image obtained by the MOJAVE program (Lister et al. 2009) on 2014 March 30 (MJD 56381) shows a compact core and a bright, linearly polarized jet feature located 1.2 mas downstream, at position angle $-68^{\circ}$. Both features have relatively high fractional polarization $(\sim 10 \%)$, and electric vectors aligned with the jet direction, at an angle of $102^{\circ}$ similar to that measured by SPOL, indicating a well-ordered transverse magnetic field. The fractional polarization level of the core feature is among the highest seen in the MOJAVE program (Lister et al. 2011).

The 2008-2015 observations of PKS $1441+25$ shown in Figure 1 reveal a brightening of the source in the radio, optical, and HE bands starting around MJD 56900. A simple Pearson test (see caveats in Max-Moerbeck et al. 2014a) applied to the radio and $\mathrm{HE}$ long-term lightcurves shows a correlation coefficient $r=0.75 \pm 0.02$, differing from zero by $5.4 \sigma$ based on the $r$-distribution of shuffled lightcurve points. Similarly, the analysis of the optical and HE lightcurves yields $r=0.89 \pm 0.02$, differing from zero by $4.8 \sigma$. The discrete correlation functions display broad, zero-centered peaks with widths of $\sim 100$ days, indicating no significant time lags beyond this time scale. During the period marked by gray dashed lines in Figure 1, observations on daily timescales from optical wavelengths to X-rays reveal fractional flux variations smaller than $25 \%$, compatible with the upper limits set by Fermi-LAT and VERITAS (30\% and $110 \%$ at the $95 \%$ confidence level, respectively). Such flux variations are small with respect to the four orders of magnitude spanned in $\nu F_{\nu}$, enabling the construction of a quasi-contemporaneous spectral energy distribution in Section 3.

\section{EMISSION SCENARIO}

The spectral energy distribution, with the X-ray-to-VHE data averaged over the active phase in 2015 April (MJD 57133-57140), is shown in Figure 2. The optical-to-Xray spectrum is well described by a power law with photon index $\Gamma=2.29 \pm 0.01$ from 2 to $30 \mathrm{keV}$, including a $10 \%$ intrinsic scatter in the fit procedure that accounts for the small-amplitude optical-to-UV variability. This spectrum suggests a single synchrotron component peaking below $2 \mathrm{eV} \sim 5 \times 10^{14} \mathrm{~Hz}$, created by an electron population of index $p=2 \Gamma-1 \sim 3.58 \pm 0.02$. As expected in FSRQs (Fossati et al. 1998), the emission of PKS $1441+25$ is dominated by the gamma-ray component, well-described by a single component peaking at $3.3_{-1.1}^{+1.8} \mathrm{GeV}$.

The detection of gamma-rays up to $200 \mathrm{GeV}$, about $400 \mathrm{GeV}$ in the galaxy's frame, suggests that the emitting region is located beyond the BLR, or else pair production would suppress any VHE flux even for a flat BLR geometry (Tavecchio \& Ghisellini 2012). The elevated radio state, correlated with the optical and HE brightening, also suggests synchrotron emission outside of the BLR where synchrotron self-absorption is smaller. The hypothesis of large-scale emission is strengthened by the week-long duration of the optical-to-gamma-ray flare. This behavior contrasts with other observations of bright FSRQs, displaying different flux variations at different wavelengths (e.g., Abdo et al. 2010), more in line with multi-component scenarios. The flare of PKS $1441+25$ appears to be one of the few events whose detailed temporal and spectral multiwavelength features are consistent with the emission of a single component beyond the BLR.

The BLR size can be derived using the estimated black-hole mass, $M_{\mathrm{BH}}=10^{7.83 \pm 0.13} M_{\odot}$ (Shaw et al. 2012), assuming 
$r_{\mathrm{BLR}} \simeq 10^{17} \mathrm{~cm} \times \sqrt{L_{\mathrm{disk}} / 10^{45} \mathrm{erg} \mathrm{s}^{-1}}$ (Kaspi et al. 2007) and an accretion disk luminosity that is a fraction $\eta=10 \%$ of the Eddington luminosity. Alternatively, $L_{\text {disk }}$ can be estimated from the BLR luminosity as $L_{\text {disk }} \simeq 10 \times L_{\mathrm{BLR}}$, with $L_{\mathrm{BLR}}=10^{44.3} \mathrm{erg} \mathrm{s}^{-1}$ (Xiong \& Zhang 2014). Both estimates yield $r_{\mathrm{BLR}} \simeq 0.03 \mathrm{pc}$, setting a lower limit on the distance between the black hole and the emitting region of $r \gtrsim 5000$ Schwarzschild radii.

We show in Figure 2 that a stationary model can reproduce the data, using the numerical code of Cerruti et al. (2013) and the EBL model of Gilmore et al. (2012). Non-stationary KleinNishina effects on electron cooling could be important for this source (Moderski et al. 2005). Nonetheless, the straight power law observed from optical to X-rays suggests that the upscattering electrons see photon energies that are low enough to stay out of the Klein-Nishina regime. This again indicates that the emission is outside of the BLR. We parametrize the electron-positron population by a fixed broken power law with indices $p_{1}=2$ and $p_{2}=3.58$ between $\gamma_{\min }=1$ and $\gamma_{\max }=7 \times 10^{5}$, with a break at $\gamma_{\text {break }}=1.2 \times 10^{4}$ (jet frame). The particle energy density is on the order of the magnetic energy density, $u_{e} / u_{B}=1.5$, with a total luminosity of $6 \times 10^{45} \mathrm{erg} \mathrm{s}^{-1}$, and an infrared photon energy density of $1.4 \times 10^{-5} \mathrm{erg} \mathrm{cm}^{-3}$ at $T=10^{3} \mathrm{~K}$ (galaxy frame), characteristic of thermal radiation from the torus. We broke the degeneracies among the parameters of the model by requiring that the minimum variability timescale in the observer frame, $t_{\mathrm{var}}=(1+z) / \delta \times R / c$, be comparable to the flux-halving timescale of about two weeks observed in $\mathrm{X}$ rays, where we have the best statistics to probe variability. The data are then modeled with an emitting region of radius $R=4 \times 10^{17} \mathrm{~cm} \sim 0.1 \mathrm{pc}$ and Doppler factor $\delta \sim 18$. The magnetic field, $B \sim 80 \mathrm{mG}$, is tangled within the emitting frame, but compressed transversely to the motion within the observer's frame, which would explain the high opticalpolarization degree, PD. Following Sasada et al. (2014), the ratio of $\mathrm{PD}$ over the maximum theoretical polarization $\Pi_{S}=(p+1) /(p+7 / 3)$ constrains the angle $\theta$ at which the emitting region is viewed. We find that the Doppler factor and the geometry of the system are well reproduced by a jet Lorentz factor $\Gamma_{\text {jet }} \sim 12$ and $\theta \sim 2.6$, which is within the jet opening angle, $\theta_{\text {jet }}=1 / \Gamma_{\text {jet }} \sim 4$. 8 .

The location of the emission can be roughly estimated assuming that the whole cross-section of the jet contributes to the radiation (Tavecchio et al. 2010), as $r \sim R / \tan \theta_{\text {jet }} \sim$ $1.5 \mathrm{pc}$, or 200,000 Schwarzschild radii. The region is not expected to be much more compact than in this model, as no fast large-amplitude variability is seen from optical to X-ray wavelengths, despite the statistics being sufficient to detect doubling timescales as short as days. The region could still be further away from the black hole if its size was only fraction of the jet cross-section.

The model parameters are similar to those obtained by MAGIC Collaboration et al. (2008), Böttcher et al. (2008), Tavecchio et al. (2011), and Barnacka et al. (2014) for other FSRQs, but a remarkably high break energy in the electron spectrum is needed to explain the optical-to-X-ray synchrotron emission. The break in the electron distribution is consistent with radiative cooling, but is pushed to higher energies due to the magnetic field being two to ten times lower than that inferred for other VHE FSRQs. The magnetic field is also in equipartition with the particle population, minimizing the energy budget required to produce the synchrotron emission. Finally, the jet Lorentz factor is two to four times smaller than that required for other VHE FSRQs, highlighting again the reasonable energetics of this scenario.

\section{EXTRAGALACTIC BACKGROUND LIGHT}

The redshift of PKS $1441+25, z=0.939$, and its detection up to $200 \mathrm{GeV}$ provide an exceptional opportunity to study the EBL.

Gamma-rays interact with EBL photons through pair production, yielding an observed spectrum that is softer than the intrinsic spectrum. Imposing a maximum intrinsic VHE hardness can then constrain the EBL intensity (Aharonian et al. 2006). In a scenario where the HE and VHE energy photons originate from the same component (but see Stern \& Poutanen 2014, for emission within the BLR), the unattenuated HE observations set an upper limit on the intrinsic hardness. Based on Section 3, we neglect any additional emission component and fit, as in Biteau \& Williams (2015), an absorbed power law with free EBL normalization, $\alpha$, to the VERITAS spectrum. We minimize

$$
\begin{aligned}
\chi^{2}= & \sum_{i=1 . . n} \frac{\left(\phi_{i}-\phi_{0} \times e^{\left.-\Gamma \log \left(E_{i} / E_{0}\right)-\alpha \tau\left(E_{i}\right)\right)^{2}}\right.}{\sigma_{\phi_{i}}^{2}} \\
& +\Theta\left(\Gamma_{\mathrm{LAT}}-\Gamma\right) \times \frac{\left(\Gamma_{\mathrm{LAT}}-\Gamma\right)^{2}}{\sigma_{\Gamma}^{2}},
\end{aligned}
$$

where $\alpha, \phi_{0}$, and $\Gamma$ are free parameters, $E_{0}$ is fixed to $120 \mathrm{GeV}$, $\left\{\phi_{i}\right\}_{i=1 . . n}$ are the fluxes measured by VERITAS at energies $\left\{E_{i}\right\}_{i=1 . . n}$, and $\Theta$ is the Heaviside function. The Fermi-LAT log-parabolic spectrum in Figure 2 shows a photon index $\Gamma_{\text {LAT }}=2.76 \pm 0.43$ at $30 \mathrm{GeV}$, where the absorption by the EBL is smaller than 5\% (Franceschini et al. 2008; Domínguez et al. 2011; Gilmore et al. 2012). We account for the systematic uncertainty on the VERITAS photon index, 0.20 , by imposing a maximum hardness with uncertainty $\sigma_{\Gamma}=0.43 \oplus 0.20=$ 0.47 , where $\oplus$ indicates a quadratic sum. We finally marginalize the equivalent likelihood, $\exp \left(-\chi^{2} / 2\right)$, over the VERITAS energy scale. The logarithm of the latter is assumed to be Gaussian, with zero mean and width 0.2 , corresponding to a $20 \%$ systematic uncertainty. Equation (1) allows for an intrinsic VHE spectrum that is softer, but not harder, than the HE spectrum. This yields an EBL normalization that is consistent with $\alpha=0$ and constrained to $\alpha<1.5$ at the $95 \%$ confidence level for the model of Gilmore et al. (2012). This result is almost independent of the choice of model (Franceschini et al. 2008; Domínguez et al. 2011).

Considering both the peak and full width at half maximum (FWHM) of the cross section integrated along the line of sight (as in Biteau \& Williams 2015, with various evolutions tested), the VERITAS observations constrain the near-ultraviolet to near-infrared EBL. Our constraint shown in Figure 3 is compatible and competitive below $1 \mu \mathrm{m}$ with other state-ofthe-art gamma-ray measurements from Ackermann et al. (2012), H.E.S.S. Collaboration et al. (2013b), and Biteau \& Williams (2015). Although $\alpha$ is compatible with zero, there is no significant tension with local constraints (see Biteau \& Williams 2015, brown and orange arrows in Figure 3), since 


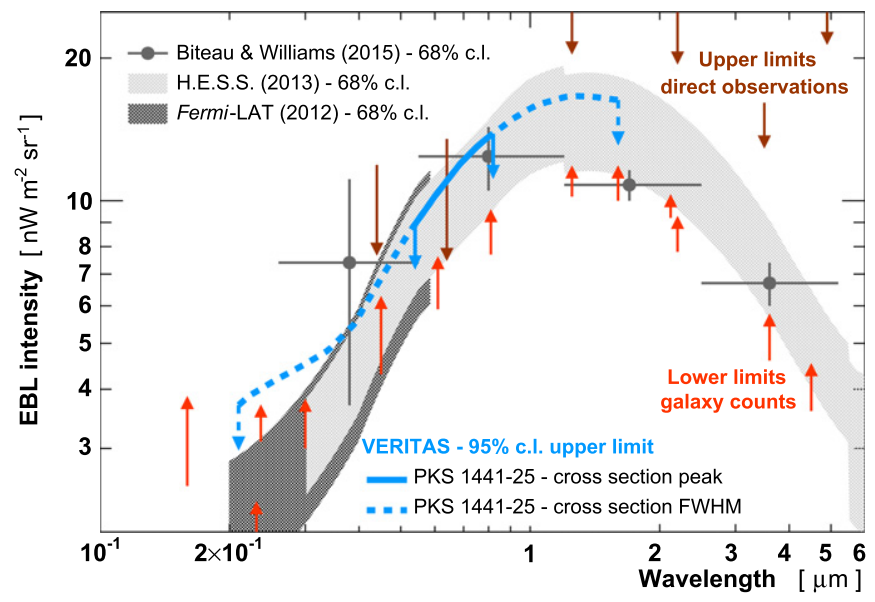

Figure 3. Near-ultraviolet to near-infrared spectrum of the EBL. The upper limit from this work is shown in blue, in regions corresponding to the peak and FWHM of the cross section $(1<\tau<2)$.

the differences are only $1.5 \sigma$ and $1.7 \sigma$ in the peak and FWHM regions, respectively.

\section{DISCUSSION}

The low energy threshold of VERITAS enabled the detection above $80 \mathrm{GeV}$ of one of the most distant VHE gamma-ray sources $(z=0.939)$, in a redshift range previously accessible only to space-borne gamma-ray observatories. We obtain stringent constraints on the EBL intensity below $1 \mu \mathrm{m}$ and conclude that galaxy surveys have resolved most, if not all, of the sources of the EBL in this region. This provides an excellent baseline for studies above $1 \mu \mathrm{m}$ where the redshifted ultraviolet emission of primordial stars could be detected (Dwek et al. 2005; Biteau \& Williams 2015).

The VHE detection of the highly polarized source PKS 1441 +25 is contemporaneous with a period of hard HE emission and of enhanced flux at all wavelengths. The correlation between the radio, optical, and HE lightcurves, unusual for this class of sources (Max-Moerbeck et al. 2014b), together with slow multiwavelength variability, suggest that the multi-band flare was produced by a single region located $\sim 10^{4}-10^{5}$ Schwarzschild radii away from the black hole, which is consistent with the VHE-gamma-ray escape condition.

PKS $1441+25$ is by far the dimmest HE emitter of all VHEdetected FSRQs listed in the 3FGL catalog. While HE activity remains a prime trigger of VHE observations, searches for new VHE-emitting quasars could also factor in radio-to-optical brightening and synchrotron-dominated X-ray emission, as reported for PKS 1441+25. These criteria will be of particular interest if applied to distant FSRQs, possibly opening a new observational window on the jets of blazars and on the transformation of the universe's light content with cosmic time.

This research is supported by grants from the U.S. Department of Energy Office of Science, the U.S. National Science Foundation and the Smithsonian Institution, and by NSERC in Canada, with additional support from NASA Swift GI grant NNX15AR38G. We acknowledge the excellent work of the technical support staff at the Fred Lawrence Whipple Observatory and at the collaborating institutions in the construction and operation of the instrument. The VERITAS Collaboration is grateful to Trevor Weekes for his seminal contributions and leadership in the field of VHE gamma-ray astrophysics, which made this study possible.

ASAS-SN thanks LCOGT, NSF, Mt. Cuba Astronomical Foundation, OSU/CCAPP and MAS/Chile for their support.

The observations at Steward Observatory are funded through NASA Fermi GI grant NNX12AO93G.

CRTS is supported by the NSF grants AST-1313422 and AST-1413600.

The OVRO 40-m monitoring program is supported in part by NASA grants NNX08AW31G and NNX11A043G, and NSF grants AST-0808050 and AST-1109911.

The National Radio Astronomy Observatory is a facility of NSF operated under cooperative agreement by Associated Universities, Inc.

This research has made use of data from the MOJAVE database that is maintained by the MOJAVE team (Lister et al. 2009).

\section{REFERENCES}

Abdo, A. A., Ackermann, M., Ajello, M., et al. 2010, Natur, 463, 919 Acero, F., Ackermann, M., Ajello, M., et al. 2015, ApJS, 218, 23 Ackermann, M., Ajello, M., Allafort, A., et al. 2012, Sci, 338, 1190 Aharonian, F., Akhperjanian, A. G., Bazer-Bachi, A. R., et al. 2006, Natur, 440, 1018

Aleksić, J., Antonelli, L. A., Antoranz, P., et al. 2011, ApJL, 730, L8 Archambault, S., Aune, T., Behara, B., et al. 2014, ApJL, 785, L16 Atwood, W. B., Abdo, A. A., Ackermann, M., et al. 2009, ApJ, 697, 1071 Barnacka, A., Moderski, R., Behara, B., Brun, P., \& Wagner, S. 2014, A\&A, 567, A113

Biteau, J., \& Williams, D. A. 2015, ApJ, 812, 60

Böttcher, M. 2008, in AIP Conf. Ser. 1085, High Energy Gamma-Ray Astronomy, ed. F. A. Aharonian, W. Hofmann, \& F. Rieger (Melville, NY: AIP), 427

Cerruti, M., Dermer, C. D., Lott, B., Boisson, C., \& Zech, A. 2013, ApJL, 771, L4

Domínguez, A., Primack, J. R., Rosario, D. J., et al. 2011, MNRAS, 410, 2556 Donea, A.-C., \& Protheroe, R. J. 2003, APh, 18, 377

Drake, A. J., Djorgovski, S. G., Mahabal, A., et al. 2009, ApJ, 696, 870

Drake, A. J., Catelan, Djorgovski, S. G., et al. 2013, ApJ, 763, 32

Dwek, E., Arendt, R. G., \& Krennrich, F. 2005, ApJ, 635, 784

Fossati, G., Maraschi, L., Celotti, A., Comastri, A., \& Ghisellini, G. 1998, MNRAS, 299, 433

Franceschini, A., Rodighiero, G., \& Vaccari, M. 2008, A\&A, 487, 837

Gehrels, N., Chincarini, G., Giommi, P., et al. 2004, ApJ, 611, 1005

Gilmore, R. C., Somerville, R. S., Primack, J. R., \& Domínguez, A. 2012, MNRAS, 422, 3189

Harrison, F. A., Craig, W. W., Christensen, F. E., et al. 2013, ApJ, 770, 103

H.E.S.S. Collaboration et al. 2013a, A\&A, 554, A107

H.E.S.S. Collaboration et al. 2013b, A\&A, 550, A4

Holder, J. 2011, Proc. ICRC, 12, 137

Jenkins, E. B., \& Savage, B. D. 1974, ApJ, 187, 243

Kalberla, P. M. W., Burton, W. B., Harmann, D., et al. 2005, A\&A, 440, 775

Kaspi, S., Brandt, W. N., Maoz, D., et al. 2007, ApJ, 659, 997

Lister, M. L., Aller, M., Aller, H., et al. 2011, ApJ, 742, 27

Lister, M. L., Cohen, M. H., Homan, D. C., et al. 2009, AJ, 138, 1874

Madau, P., \& Pozzetti, L. 2000, MNRAS, 312, L9

MAGIC Collaboration et al. 2008, Sci, 320, 1752

Max-Moerbeck, W., Hovatta, T., Richards, J. L., et al. 2014b, MNRAS, 445, 428

Max-Moerbeck, W., Richards, J. L., Hovatta, T., et al. 2014a, MNRAS, 445,437

Mirzoyan, R. 2015, ATel, 7416, 1

Moderski, R., Sikora, M., Coppi, P. S., \& Aharonian, F. 2005, MNRAS, 363,954

Pacciani, L. 2015, ATel, 7402, 1

Richards, J. L., Max-Moerbeck, W., Pavlidou, V., et al. 2011, ApJS, 194, 29 Roming, P. W. A., Kennedy, T. E., Mason, K. O., et al. 2005, SSRv, 120, 95

Sasada, M., Uemura, M., Fukuzawa, Y., et al. 2014, ApJ, 784, 141

Schmidt, G. D., Stockman, H. S., \& Smith, P. S. 1992, ApJL, 398, L57

Shappee, B. J., Prieto, J. L., Grupe, D., et al. 2014, ApJ, 788, 48

Shaw, M. S., Romani, R. W., Cotter, G., et al. 2012, ApJ, 748, 49 
Sitarek, J., Becerra-González, J., Dominis Prester, D., et al. 2015, arXiv: 1508.04580

Stern, B. E., \& Poutanen, J. 2014, ApJ, 794, 8

Tavecchio, F., Becerra-González, J., Ghisellini, G., et al. 2011, A\&A, 534, A86

Tavecchio, F., \& Ghisellini, G. 2012, arXiv:1209.2291
Tavecchio, F., Ghiselleni, G., Bonnoli, G., \& Ghirlanda, G. 2010, MNRAS, 405, L94

Vaughan, S., Edelson, R., Warwich, R. S., \& Uttley, P. 2003, MNRAS, 345,1271

Xiong, D. R., \& Zhang, X. 2014, MNRAS, 441, 3375 